\title{
Gestation and breastfeeding in schistosomotic mice differentially alters the expression of histone deacetylases (HDACs) in adult offspring
}

\author{
Gabriela Calixto Ribeiro de Holanda', Fabrício Oliveira Souto', \\ Maria da Conceição Silva', Virgínia Maria Barros de Lorena ${ }^{2}$, Vlaudia Maria Assis Costa1, \\ Monica Camelo Pessôa de Azevedo Albuquerque ${ }^{1}$, Valdênia Maria Oliveira de Souza ${ }^{1 /+}$, \\ José Luiz de Lima Filho'
}

1Universidade Federal de Pernambuco, Laboratório de Imunopatologia Keizo Asami, Recife, PE, Brasil

2Fundação Oswaldo Cruz-Fiocruz, Instituto de Pesquisas Aggeu Magalhães, Recife, PE, Brasil

BACKGROUND Breastfeeding or gestation in schistosomotic mothers can cause long-term alterations in the immune response of offspring.

OBJECTIVES Evaluate the expression of histone deacetylases (HDACs) (all classes), the production of cytokines by $\mathrm{T}$ and $\mathrm{B}$ lymphocytes and macrophages, and the frequency of $\mathrm{CD} 4{ }^{+} \mathrm{CD} 25^{+} \mathrm{FoxP} 3^{+}$-cells in adult offspring born and/or suckled by schistosomotic mothers.

METHODS We harvested splenocytes from offspring born to (BIM), suckled by (SIM), or born to/suckled by (BSIM) schistosomotic mothers and animals from noninfected mothers (Control) at seven-weeks old and cultured them with/without Concanavalin A. HDAC expression was evaluated by real-time quantitative polymerase chain reaction (qPCR), and cytokines and membrane markers were evaluated by fluorescence-activated cell sorting (FACS).

FINDINGS Compared to Control, BIM mice showed increased expression of HDAC9 and frequency of CD4 ${ }^{+}$IL- $10^{+}$-cells. The SIM group had increased expression of HDAC1, HDAC2, HDAC6, HDAC7, HDAC10, Sirt2, Sirt5, Sirt6, and Sirt7. The BSIM group only had increased HDAC10 expression. The SIM and BSIM groups exhibited decreased frequencies of CD $4^{+}$IL- $4^{+}$-cells and $\mathrm{CD} 4^{+} \mathrm{CD} 25^{+} \mathrm{FoxP} 3^{+}$-cells, along with a higher frequency of CD14 $4^{+} \mathrm{LL}-10^{+}$-cells and an increase in CD $45 \mathrm{R} / \mathrm{B} 220^{+} \mathrm{IL}-10^{+}$-cells. The BSIM group also showed a high frequency of CD4 $4^{+} \mathrm{IL} 10^{+}$-cells.

MAIN CONCLUSIONS Breastfeeding induced the expression of HDACs from various classes involved in reducing inflammatory responses. However, gestation enhanced the expression of a single HDAC and breastfeeding or gestation appears to favour multiple IL-10-dependent pathways, but not cells with a regulatory phenotype.

Key words: breastfeeding - epigenomics - histone deacetylases - pregnancy - schistosomiasis

A high prevalence of chronic schistosomiasis in pregnant women and women of childbearing age has been reported, ${ }^{(1)}$ and the effects of maternal infection have raised questions regarding the immunity of the offspring.

It is known that the immunological status of schistosomotic mothers can induce long-term alterations in the immune response of the offspring. ${ }^{(2,3,4,5)}$ An experimental study on the effects of gestation and breastfeeding in infected mothers, separately, showed that gestation in these mothers led to potential immunosuppression in adult offspring, with elevated production of IL-10 and lower levels of anti-ovalbumin (OA) antibodies. ${ }^{(2)}$ In addition, offspring born to infected mothers had a lower frequency of B lymphocytes, and the capacity for antigen presentation by CD11c+ cells was partially impaired. ${ }^{(3)}$ In contrast, it has also been observed that adult mice pre-

doi: 10.1590/0074-02760190366

Financial support: FACEPE, CNPq.

+ Corresponding author: valdenia.souza@gmail.com

(1) https://orcid.org/ 0000-0002-4976-009x

Received 02 October 2019

Accepted 09 December 2019 viously breastfed by schistosomotic mothers exhibited improvement in the production of anti-OA antibodies ${ }^{(2)}$ and in the antigen presentation ability of B lymphocytes through an increase in surface frequency of CD40+/ CD80+ in these cells. ${ }^{(3)}$ However, whether these alterations in the immune response of adult offspring from infected mothers are due to epigenetic changes from the perinatal period remains unclear.

Studies have correlated post-transcriptional changes in the chromatin, through histone acetylation/deacetylation, with the immune response. ${ }^{(6,7,8)}$ In an experimental study on antigen presenting cells (APCs), it was demonstrated that histone deacetylase (HDAC)6 is required for transcriptional activation of IL-10 gene expression in macrophages and dendritic cells through activation of STAT3 ${ }^{(6)}$ Another study using pancreatic beta cell lines showed that knockdown of HDAC1 increased IFN- $\gamma$ induced STAT1 phosphorylation. ${ }^{(7)}$ Kosciuczuk et al. ${ }^{(8)}$ showed that deacetylation of cyclin-dependent kinase 9 induced by Sirtuin 2 promotes STAT1 phosphorylation during type I interferon responses.

In addition, it has been demonstrated that the role of epigenetic markers can be remodelled during the perinatal period, and may trigger lasting influences on the epigenome of the offspring. ${ }^{(9)}$ Mice prenatally adminis- 
tered with Acinetobacter lwoffii F78 displayed increased acetylation of histone $\mathrm{H} 4$ in the interferon (IFN)- $\gamma$ gene in their offspring, and conferred protection against asthma after challenge with OA, which is associated with positive regulation of IFN- $\gamma$ production. ${ }^{(10)}$ Song et al. ${ }^{(11)}$ found that offspring from mothers with peanut allergy had elevated IgE-specific levels, high levels of histamine and resultant increased production of Th2 cytokines, and reduction of DNA methylation at $\mathrm{CpG}$ sites of the IL-4 gene promoter after sensitisation.

Histone acetylation is the most commonly studied epigenomic alteration, for stimulation of transcription, and in turn, is reversibly regulated by the balance between the activity of histone acetyltransferases (HATs) and HDACs. ${ }^{(12)}$ HDACs have been classified as class I (HDAC1, HDAC2, HDAC3 and HDAC8), class IIa (HDAC4, HDAC5, HDAC7, and HDAC9), class IIb (HDAC6 and HDAC10), class III (SIRT1 to SIRT7), and class IV (HDAC11) ${ }^{(13)}$ and are increasingly studied due to their interference in the pathways of mechanisms associated with the pathogenesis of various cancers and other inflammatory diseases. ${ }^{(14,15,16)}$

Although research that relates epigenetic alterations to the maternal-foetal relationship can be found, there are no studies that report the effects of gestation and/or breastfeeding on the expression of HDACs, and the implications for the immune system of offspring from schistosomotic mothers. To investigate, we have evaluated whether the expression of enzymes involved in chromatin remodelling through histone deacetylation can be altered due to gestation or breastfeeding from Schistosoma mansoni-infected mothers. Our results could aid in the discovery of therapeutic targets that improve the immunity of individuals who previously contacted immunological factors resulting from infection during perinatal period.

\section{MATERIALS AND METHODS}

Animals and maternal infection - Four-week-old Swiss Webster female mice were infected subcutaneously (s.c.) with 20 S. mansoni cercariae, strain São Lourenço da Mata (SLM). On the 45th day, infection was confirmed by the Kato-Katz method. ${ }^{(17)}$ On the 60 th day post-infection (dpi), oestrus was synchronised among mice via the administration of 5 i.u. $(100 \mu \mathrm{L})$ of equine chorionic gonadotrophin hormone, followed by an additional injection with 5 i.u. $(100 \mu \mathrm{L})$ of human chorionic gonadotrophin $48 \mathrm{~h}$ later. Females were housed with male mice at a 1:1 ratio, and successful mating was confirmed by presence of a vaginal plug. The same procedure was performed in noninfected females, and sevenweek-old male offspring were taken for the experimental and control groups. The mice were housed in the animal care facility at the Aggeu Magalhães Institute (IAM), Oswaldo Cruz Foundation (Fiocruz), municipality of Recife, State of Pernambuco, Brazil.

Adoptive nursing and study groups - Immediately after birth, new-born mice from S. mansoni-infected or noninfected mothers were rehoused with mothers from the opposite group. After adoptive nursing, offspring born from infected mothers (BIM) were suckled by noninfected mothers, and offspring from noninfected moth- ers were suckled by infected mothers (SIM). A separate group was born from and suckled by schistosomotic mothers (BSIM). Animals born from noninfected females were suckled by their mothers (Control).

Cell culture - Spleens from each animal (sevenweeks-old) were harvested after euthanasia by cervical dislocation. Cell suspensions were prepared in RPMI1640 (Sigma-Aldrich, St. Louis, USA) supplemented with HEPES $(10 \mu \mathrm{M}), 2$-mercaptoethanol $(0.05 \mu \mathrm{M}), 216$ $\mathrm{mg}$ of L-glutamine/L, gentamicin $(50 \mathrm{mg} / \mathrm{L})$, and $5 \%$ of foetal bovine serum (FBS) (Sigma-Aldrich, St. Louis, USA). Cells from each group $(n=8-10)$ were cultivated at a final concentration of $2 \times 10^{7}$ cells $/ \mathrm{mL}$ in tissue culture plates (Costar Culture Plates, City, USA) and stimulated with concanavalin-A (Con-A) $(5 \mu \mathrm{g} / \mathrm{mL})$, or without antigenic stimulus (Basal), at $37^{\circ} \mathrm{C}$ in $5 \% \mathrm{CO}_{2}$. Cultured cells were harvested after $24 \mathrm{~h}$ and assayed for immunophenotyping and real-time quantitative polymerase chain reaction (qPCR).

Flow cytometry analyses - $5 \mu \mathrm{L}$ of Golgi Stop (per 2 $\times 10^{7}$ cells) were added to each well containing splenic cells under different stimuli, then the cells were vortexed and returned to the $\mathrm{CO}_{2}$ incubator at $37^{\circ} \mathrm{C}$ for four additional hours. Spleen cells were subjected to double-labelling with fluorochrome-labelled antibody solutions at a concentration of $0.5 \mathrm{mg} / 10^{6}$ cells: FITC anti-mouse CD4, and PE anti-mouse IL-4, APC anti-mouse IFN- $\gamma$, PE anti-mouse IL-10, or PerCP-Cy-5.5 anti-mouse IL-2; FITC anti-mouse CD4, PE anti-mouse CD25, and APC anti-mouse FoxP3; FITC anti-mouse CD45R (B220) or FITC anti-mouse CD14, and PE anti-mouse IL-10 (BD Biosciences Pharmingen). After staining, preparations were washed with phosphate-buffered saline (PBS) containing azide $(0.1 \%)$ and FBS $(3 \%)$. After centrifugation, the cell pellet was resuspended in PBS with paraformaldehyde $(0.5 \%)$ and maintained at $4^{\circ} \mathrm{C}$ until data acquisition, which was performed using a FACSCalibur (BD-Pharmingen, New Jersey, USA) flow cytometer and acquisition of a minimum 50,000 lymphocytes or 5,000 monocytes. The frequency of positive cells was analysed using FlowJo software, with quadrant gating set based on negative populations and isotype controls. A descriptive analysis of the frequency of cells in the upper right quadrant (double-positive cells) was performed. Distinct gating strategies were used to analyse each subpopulation of cells (Fig. 1). T lymphocyte subpopulations were first selected as CD4 high cells on FL1/anti-CD4-FITC versus laser side-scatter (SSC) dot plots (Fig. 1A). Following this gating procedure, a second gate was set using FL1/anti-CD4-FITC versus FL2/ anti-CD25-PE; then, a third gate was established to generate representative 2-dimensional graphics using FL1/ anti-CD4-FITC versus FL4/anti-FoxP3-APC to identify triple staining for CD4+CD25+FoxP3+ (Fig. 1B). The frequency of cytokine-expressing cells was further determined on FL1/anti-CD4-FITC versus FL2/anti-IL10PE or anti-IL4-PE, FL3/anti-IL2-Percp-Cy-5.5 or FL4/ anti-IFN- $\gamma$-APC dot plots by quadrant statistic measurements, and expressed as percentage of cytokine T CD4+ lymphocyte (Fig. 1C). B Cells and monocytes were first 
selected on CD45-high or CD14-high cells using FL1/ anti-CD45 or CD14-FITC versus SSC dot plots, and the frequency of IL-10 producing cells was subsequently determined using FL1/CD45-FITC or FL1/CD14-FITC versus anti-IL10-PE dot plots and quadrant statistic measurements (Fig. 1D-E).

The results are expressed as the median frequency of cells from each group \pm standard error.

$q P C R$ analysis - Total RNA from splenic cells was extracted using the ReliaPrep ${ }^{\mathrm{TM}}$ RNA Cell Miniprep System Kit (Promega, Madison, WI) according to the man-
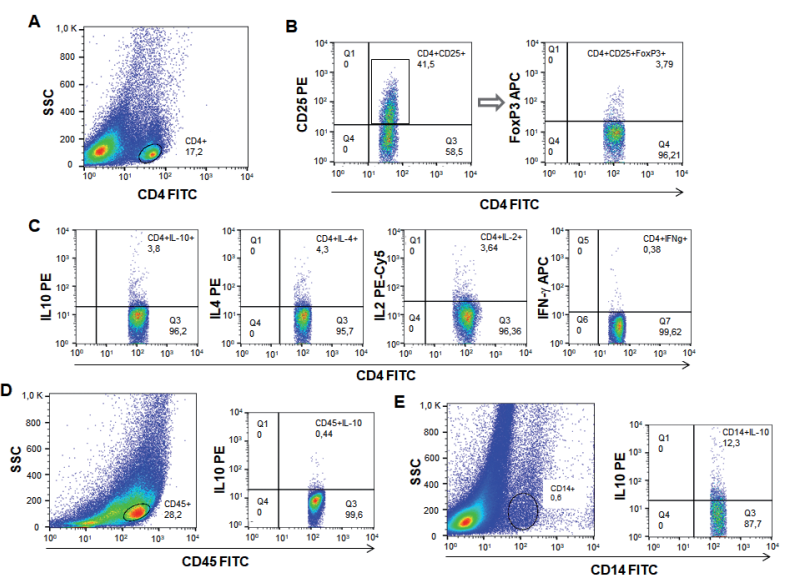

Fig. 1: representation of the gating strategy used to analyse the different subpopulations of cells. FL1/anti-CD4-FITC versus laser side-scatter (SSC) dot plot (A), CD4+CD25+FoxP3+ cells dot plots (B), cytokine producing T CD4+ lymphocytes dot plots (C), IL-10 producing CD45R/B220+ cells dot plot (D) and IL-10 producing $\mathrm{CD} 14+$ cells dot plot (E).
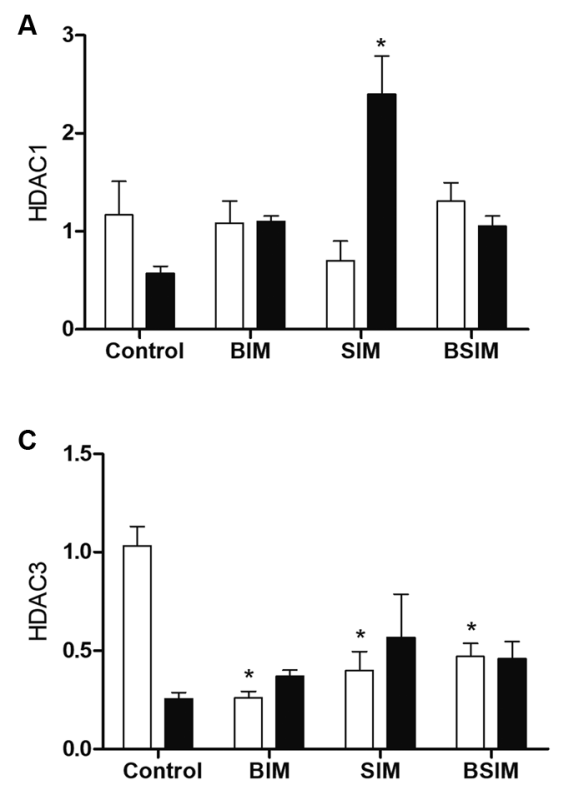

ufacturer's instructions. Complementary DNA (cDNA) was generated with the QuantiTect Reverse Transcription Kit (Qiagen, Hilden, Germany). Quantitative PCR was performed using SYBR Green master mix (Applied Biosystems, Foster City, CA, USA) on the 7500 Real Time System (Applied Biosystems Foster City, CA, USA) machine. Results were normalised to the housekeeping gene $\beta$-Actin. Relative expression levels were calculated using $2^{\Delta \Delta C t}$. Primers were designed using Primer3Plus software, and the sequences are described in Table.

Statistical analysis - Results were subjected to Barlett's test to verify whether the distribution of the data was normal. After verifying that the results did not follow a normal distribution, the Kruskal-Wallis test was used, followed by Dunn's multiple comparison test when statistical significance was shown. For statistical analysis, we used GraphPad Prism v.5.0 (GraphPad Software, San Diego, CA, USA) and findings were considered significant at $p<0.05$. All procedures were performed in triplicate to evaluate reproducibility, and images refer to one representative of at least three independent studies.

Ethics - The animal protocol was approved by the Ethical Commission on Animal Use of the Fiocruz $(113 / 2017)$ and is in accordance with the Ethical Principles in Animal Research adopted by the Brazilian College of Animal Experimentation.

\section{RESULTS}

Relative expression of HDAC in animals born and/ or breastfed from schistosomotic mothers - To verify the epigenetic profile of the animals born and/or breastfed from schistosomotic mothers, real time qPCR was performed on spleen cells cultured for $24 \mathrm{~h}$ in absence
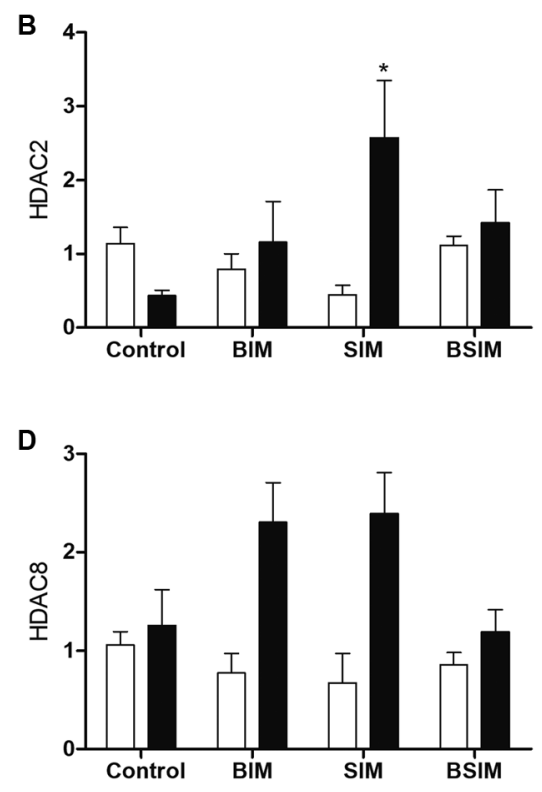

Fig. 2: relative expression of class I histone deacetylases (HDACs). HDAC1 (A), HDAC2 (B), HDAC3 (C) and HDAC8 (D) in splenocytes from Swiss Webster mice (seven weeks) born (BIM), breastfed (SIM), or born and breastfed (BSIM) in infected mothers, or uninfected mothers (Control), cultured for $24 \mathrm{~h}$ in the presence of mitogen (Concanavalin A) $(5 \mu \mathrm{g} / \mathrm{mL}$, black bar) or with culture medium (BASAL, white bar). The relative expression was verified by real-time quantitative polymerase chain reaction (qPCR). The results represent the median and standard error for 8-10 animals/group. " $\mathrm{p}<0.05$ compared to control group. 
TABLE

Primers used in quantitative real-time polymerase chain reaction (qPCR)

\begin{tabular}{|c|c|c|c|c|}
\hline Target gene (Accession number) & $\begin{array}{c}\text { Sequence 5'-3' } \\
\text { (Forward and reverse) }\end{array}$ & $\mathrm{Tm}$ & CG \% & Amplicon length (bp) \\
\hline \multirow{2}{*}{ HDAC1 (NM_008228.2) } & CCGGTTAGGTTGCTTCAATC & 59.6 & 50 & \multirow{2}{*}{118} \\
\hline & AACATTCCGGATGGTGTAGC & 59.8 & 50 & \\
\hline \multirow{2}{*}{ HDAC2 (NM_008229.2) } & TATTATGGCCAGGGTCATCC & 59.6 & 50 & \multirow{2}{*}{119} \\
\hline & TCAGCAGTGGCTTTATGAGG & 59 & 50 & \\
\hline \multirow{2}{*}{ HDAC3 (NM_010411.2) } & ATGCAGGGTTTCACCAAGAG & 60.1 & 50 & \multirow{2}{*}{117} \\
\hline & TGTTGCTCCTTGCAGAGATG & 60.1 & 50 & \\
\hline \multirow{2}{*}{ HDAC4 (NM_207225.2) } & CCGCCAGCAGTTTTAAAGTC & 59.9 & 50 & \multirow{2}{*}{92} \\
\hline & ACCGAATGGAGATGCTCAAC & 60.1 & 50 & \\
\hline \multirow{2}{*}{ HDAC5 (NM_001077696.1) } & ACTTTCCCCTCCGTAAAACG & 60.3 & 50 & \multirow{2}{*}{116} \\
\hline & AACAGTGCCATCCTTTCGAC & 60.1 & 50 & \\
\hline \multirow{2}{*}{ HDAC6 (NM_010413.3) } & ATCTCAGCTGGCTTTGATGC & 60.5 & 50 & \multirow{2}{*}{116} \\
\hline & ATAATACGGCCACCAGCAAG & 60.0 & 50 & \\
\hline \multirow{2}{*}{ HDAC7 (NM_001204275.1) } & ATGATGGCCTGGAACATAGG & 59.8 & 50 & \multirow{2}{*}{75} \\
\hline & GATGCTGCTGCAGAGAAATG & 59.7 & 50 & \\
\hline \multirow{2}{*}{ HDAC8 (NM_027382.4) } & AGGGAATCTGAAGCATGTGG & 60.1 & 50 & \multirow{2}{*}{131} \\
\hline & CAAATTTCCCCTGCAGTCAC & 60.5 & 50 & \\
\hline \multirow{2}{*}{ HDAC9 (NM_001271386.1) } & TTTGAGGTGGCAGAATCCTC & 60.2 & 50 & \multirow{2}{*}{106} \\
\hline & GAGCTGAAGCCTCATTTTCG & 60.1 & 50 & \\
\hline \multirow{2}{*}{ HDAC10 (NM_199198.2) } & AACAGGAGCTGTGCACAATG & 59.9 & 50 & \multirow{2}{*}{143} \\
\hline & TCCTCTGCAGCCCATATTTC & 60.2 & 50 & \\
\hline \multirow{2}{*}{ HDAC11 (NM_144919.2) } & TGATGGGGTTGAACACTGAG & 59.5 & 50 & \multirow{2}{*}{128} \\
\hline & AGCAGCCCCTTAAAAACTCC & 59.7 & 50 & \\
\hline \multirow{2}{*}{ Sirtl (NM_019812.3) } & GCCCTCAATTTCTGTTCTGC & 59.8 & 50 & \multirow{2}{*}{150} \\
\hline & TTTTGAGTGCTCCAGACACG & 60.0 & 50 & \\
\hline \multirow{2}{*}{ Sirt2 (NM_019812.3) } & ACGGCTGCTCATTAACAAGG & 60.3 & 50 & \multirow{2}{*}{88} \\
\hline & GTCAAAATCCATGCCACCTC & 60.3 & 50 & \\
\hline \multirow{2}{*}{ Sirt3 (NM_001177804.1) } & CATATGGGCTGATGTGATGG & 59.8 & 50 & 141 \\
\hline & AGATCTGCCAAAGCGAAGTC & 59.6 & 50 & 141 \\
\hline Sirt4 (NMM 0011676011$)$ & CGAGCAAAAGCTCCCAATAG & 60.0 & 50 & 145 \\
\hline SIrt4 (N101_00110/091.1) & TTCCAGCCTTTGGACATCAG & 61.2 & 50 & 143 \\
\hline Sirt5 (NM 1788483$)$ & CCAGCTTTAGCAGGAAAAGG & 59.1 & 50 & 120 \\
\hline Sirts (NIM_1/8848.3) & CCAGGTTTTCTCCAAACCAC & 59.4 & 50 & 139 \\
\hline Sirt6 (NM 1815863$)$ & TGTCCAACACAGCTCCTTTC & 58.9 & 50 & 07 \\
\hline Sirto (NIM_181586.3) & CTTCCACATGTGTGGGATTC & 58.8 & 50 & 91 \\
\hline Sirt7 (NM 0013634391$)$ & AGCTTCGGGATACCATTGTG & 60 & 50 & 104 \\
\hline SIrt/(NIV__001503439.1) & CAGGATTGTGTCTGCTTTGC & 59.4 & 50 & 104 \\
\hline & TTGCTGACAGGATGCAGAAG & 61.1 & 50 & 14 \\
\hline B-Actın (NM_007393.5) & TGATCCACATCTGCTGGAAG & 59.8 & 50 & $14 /$ \\
\hline
\end{tabular}

bp: base pair; CG: cytosine and guanine content; HDAC: histone deacetylase 1-11; Sirt: Sirtuin 1-7; Tm (melting temperature) was calculated at default settings of $0.25 \mu \mathrm{M}$ oligo concentration and $50 \mu \mathrm{M} \mathrm{Na}$. Primers were designed using Primer3Plus software (http://www.bioinformatics.nl/cgi-bin/primer3plus/primer3plus.cgi). 
A

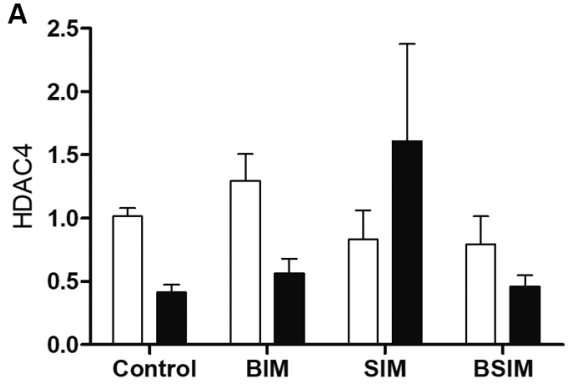

C

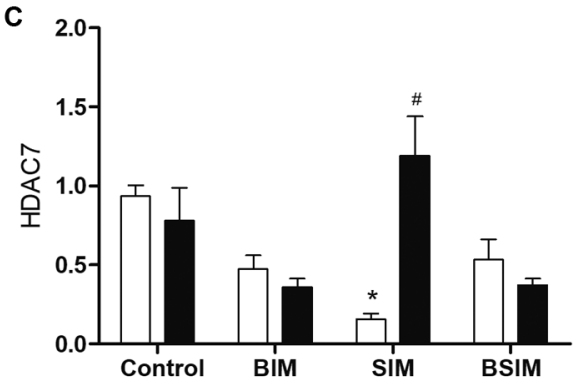

B
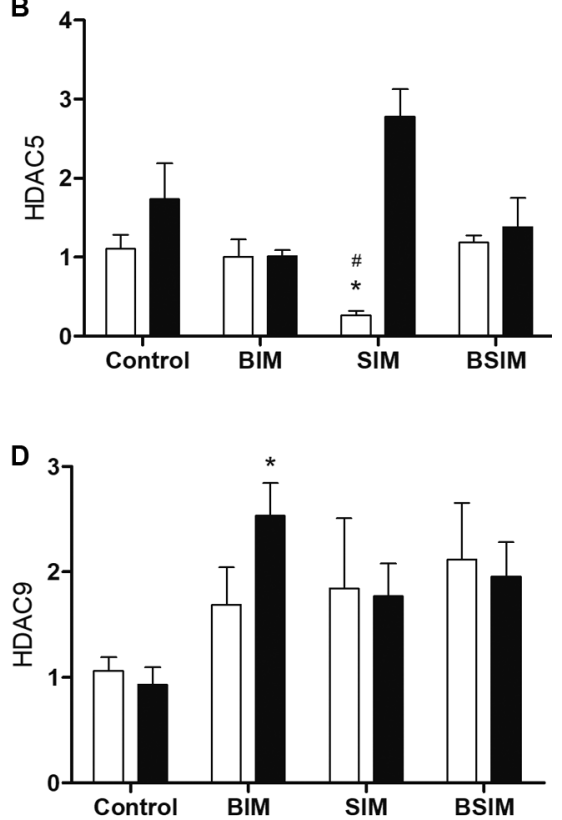

Fig. 3: relative expression of class IIa histone deacetylases (HDACs). HDAC4 (A), HDAC5 (B), HDAC7 (C) and HDAC9 (D) in splenocytes from Swiss Webster mice (seven weeks) born (BIM), breastfed (SIM), or born and breastfed (BSIM) in infected mothers, or uninfected mothers (Control), cultured for $24 \mathrm{~h}$ in the presence of mitogen (Concanavalin A) ( $5 \mu \mathrm{g} / \mathrm{mL}$, black bar) or with culture medium (BASAL, white bar). The relative expression was verified by real-time quantitative polymerase chain reaction (qPCR). The results represent the median and standard error for 8-10 animals/group. " $\mathrm{p}<0.05$ compared to control group. ${ }^{*} \mathrm{p}<0.05$ compared to BIM and BSIM groups.
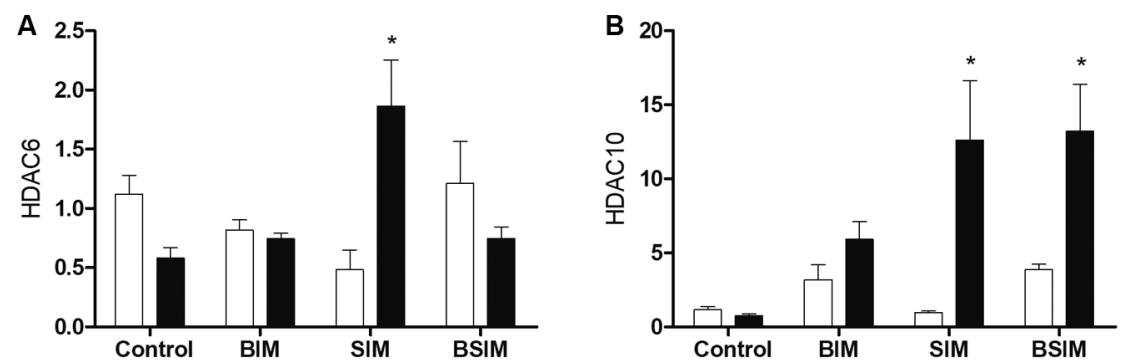

Fig. 4: relative expression of class IIb histone deacetylases (HDACs). HDAC6 (A) and HDAC10 (B) in splenocytes from Swiss Webster mice (seven weeks) born (BIM), breastfed (SIM), or born and breastfed in (BSIM) infected mothers, or uninfected mothers (Control), cultured for $24 \mathrm{~h}$ in the presence of mitogen (Concanavalin A) $(5 \mu \mathrm{g} / \mathrm{mL}$, black bar) or with culture medium (BASAL, white bar). The relative expression was verified by real-time quantitative polymerase chain reaction (qPCR). The results represent the median and standard error for 8-10 animals/ group. " $\mathrm{p}<0.05$ compared to control group.

(basal) or presence of mitogenic stimulus (ConA). For class I HDAC, the basal relative expressions of HDAC1 and HDAC2 were not altered, but with mitogenic stimulus, the group of animals which were breastfed only (SIM) exhibited increased expression compared to the Control (Fig. 2A-B). The groups BIM, SIM, and BSIM had decreased basal relative expression of HDAC3, but with mitogenic stimulus the relative expression was similar to Control (Fig. 2C). There was no difference in the relative expression of HDAC8 either at baseline or with mitogenic stimulation (Fig. 2D).

Class IIa HDACs were analysed, and we saw that there was no difference in HDAC4 expression (Fig. 3A). The relative expression of HDAC5 in the SIM group was lower compared to Control, BIM and BSIM at baseline, but there was no difference among the groups under mito- genic stimulus (Fig. 3B). For HDAC7 the SIM group had lower expression than Control at baseline, while under mitogenic stimulus the SIM group had increased expression compared to the BIM and BSIM groups (Fig. 3C). Regarding HDAC9, there was no difference at baseline, but the BIM group showed a higher relative expression compared to Control under mitogenic stimulus (Fig. 3D).

Regarding HDACs 6 and 10 (class IIb), there was similar expression among all groups, and did not differ significantly from Control. However, in response to mitogenic stimulus, the expression of HDAC6 and HDAC10 was increased in the SIM group, and HDAC10 was increased in the BSIM group (Fig. 4A-B).

Among sirtuins (class III), the expressions of Sirt1, Sirt3, and Sirt4 were found to be similar among all groups under the culture conditions used (Fig. 5A-C). 
Sirt2 and Sirt5 did not show any differences in the basal groups, but increased expression was observed in the SIM group compared to Control under mitogenic stimulation (Fig. 5D-E). Fig. 5F shows that compared to Control, the expression of Sirt6 in the SIM group was lower at baseline. However, under mitogenic stimulus, there was a significant increase in the expression of Sirt6 in all experimental groups (BIM, SIM, and BSIM). Although there was no baseline difference for Sirt7, the SIM group had increased expression with mitogenic stimulus compared to the Control, BIM, and BSIM groups (Fig. 5G).

Class IV is composed only of HDAC11 which in this study showed no differences compared to Control, but the SIM group had higher relative expression compared to BIM under mitogenic stimulus (Fig. 6).

Intracellular cytokines in $T$ and $B$ lymphocytes, and monocytes, and the frequency of regulatory T lymphocytes in animals born and/or breastfed - Cytokine producing $\mathrm{T}$ lymphocytes were observed by labelling with CD4+/IL-4+, CD4+/IFN- $\gamma+$, CD4+/IL-10+, or CD4+/
IL-2+, while B lymphocytes and monocytes were labelled with anti-CD45R/B220+ and anti-CD14+ antibodies, respectively, together with anti-IL-10+. T lymphocytes with a regulatory profile were evaluated by triple labelling $\mathrm{CD} 4+\mathrm{CD} 25+\mathrm{FoxP} 3+$. Frequencies were evaluated with mitogenic stimulus (ConA) and without (basal). Compared to the Control group, the frequency of CD4+/IL-4+ cells under basal conditions and mitogenic stimulation was lower in the SIM and BSIM groups (Fig. 7A). IL-10 production by CD4+ cells was higher in the BIM and BSIM groups under mitogenic stimulation (Fig. 7B). There were no differences among groups when the frequencies of CD4+IL-2+ and CD4+/IFN- $\gamma+$ cells (Fig. 7C-D) were analysed.

Regarding IL-10 production by B lymphocytes (Fig. 7E), it was slightly higher in the SIM and BSIM groups in response to mitogen. The SIM and BSIM groups also had an increased frequency of CD14+IL-10+ in comparison to the Control and BIM groups both at baseline and in response to mitogen (Fig. 7F).
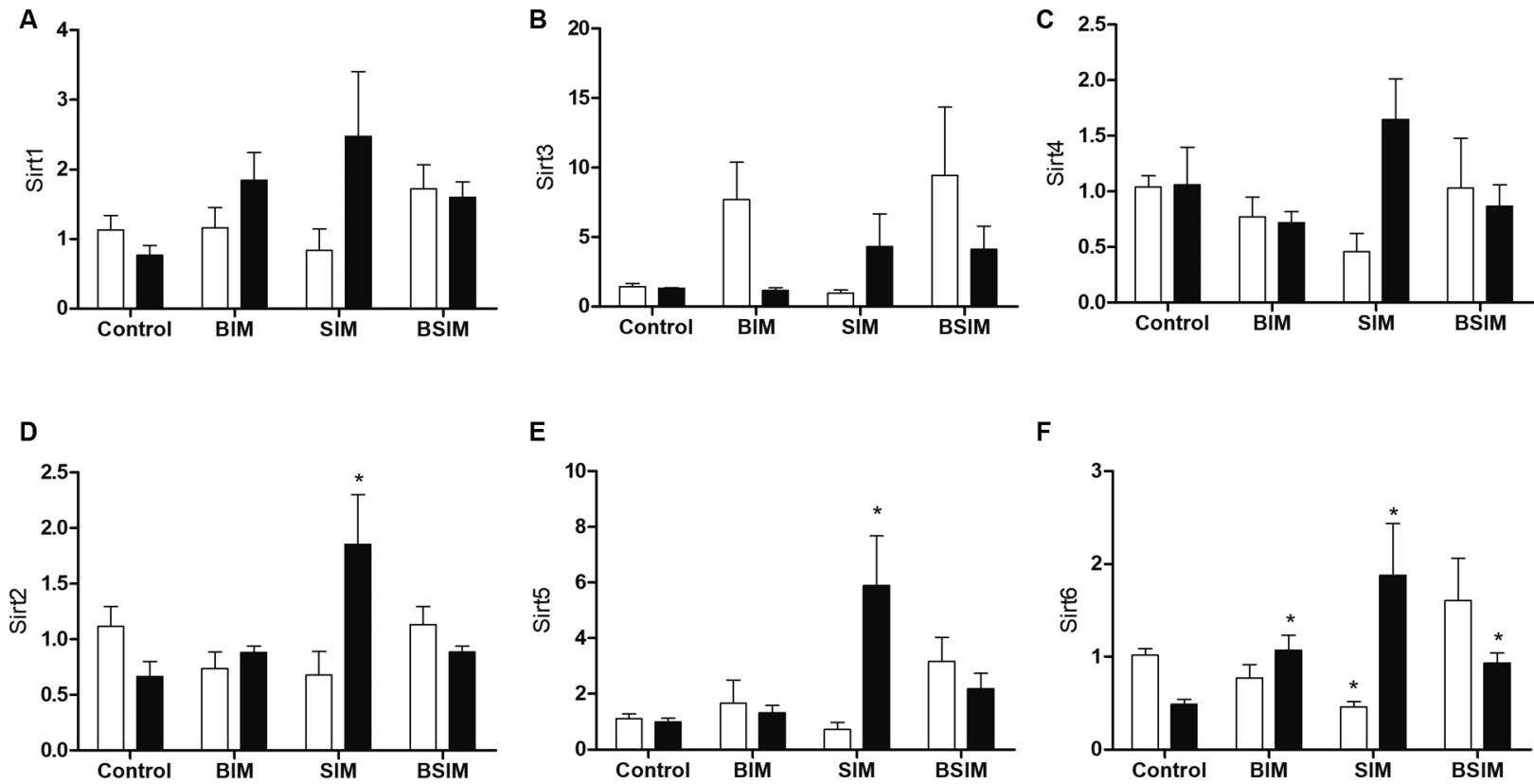

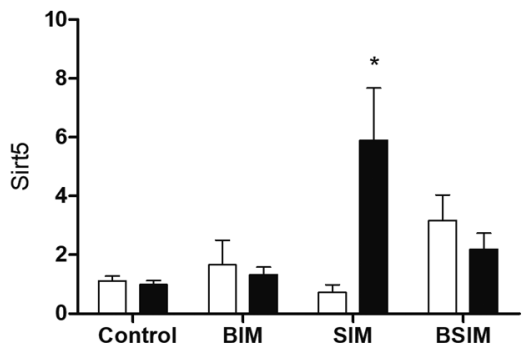

$\mathbf{F}$

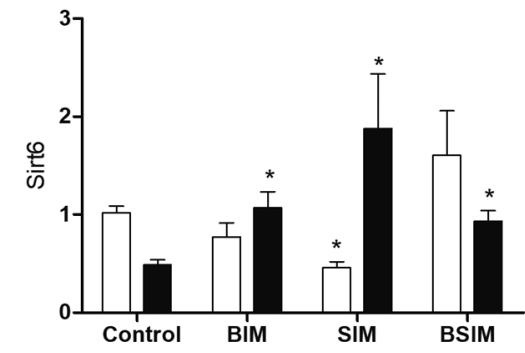

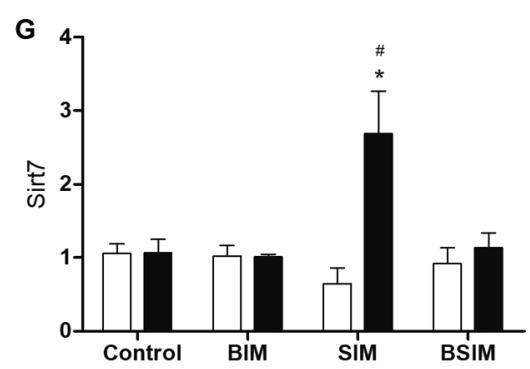

Fig. 5: relative expression of class III histone deacetylases (HDACs). Sirt1 (A), Sirt3 (B), Sirt4 (C), Sirt2 (D), Sirt5 (E), Sirt6 (F) and Sirt7 (G) in splenocytes from Swiss Webster mice (seven weeks) born (BIM), breastfed (SIM) or born and breastfed (BSIM) in infected mothers, or uninfected mothers (Control), cultured for $24 \mathrm{~h}$ in the presence of mitogen (Concanavalin A) $(5 \mu \mathrm{g} / \mathrm{mL}$, black bar) or with culture medium (BASAL, white bar). The relative expression was verified by real-time quantitative polymerase chain reaction (qPCR). The results represent the median and standard error for 8-10 animals/group. " $\mathrm{p}<0.05$ compared to control group. ${ }^{*} \mathrm{p}<0.05$ compared to BIM and BSIM groups. 


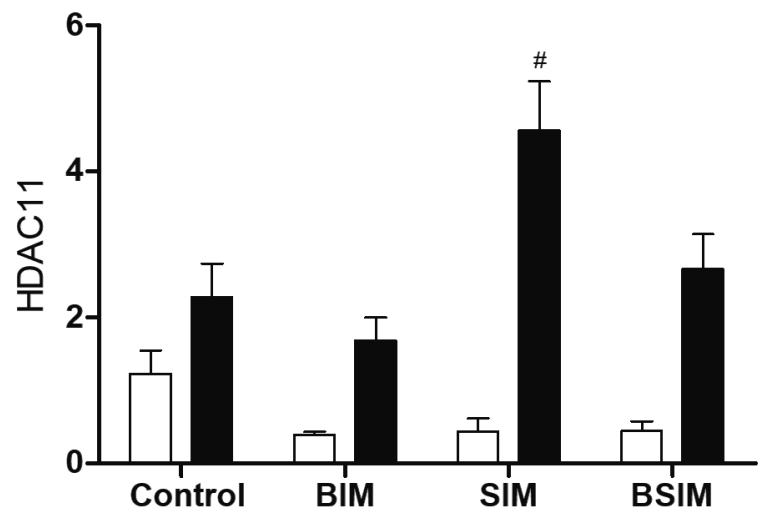

Fig. 6: relative expression of class IV histone deacetylases (HDACs). HDAC11 in splenocytes from Swiss Webster mice (seven weeks) born (BIM), breastfed (SIM) or born and breastfed(BSIM) in infected mothers, or uninfected mothers (Control), cultured for $24 \mathrm{~h}$ in the presence of mitogen (Concanavalin A) $(5 \mu \mathrm{g} / \mathrm{mL}$, black bar) or with culture medium (BASAL, white bar). The relative expression was verified by real-time quantitative polymerase chain reaction (qPCR). The results represent the median and standard error for 8-10 animals/group.
For cells expressing CD4+CD25+FoxP3+, the SIM and BSIM groups exhibited decreased frequency relative to the Control and BIM groups, with and without mitogenic stimulus (Fig. 7G).

\section{DISCUSSION}

Maternal infection by S. mansoni can alter the degree of immune competence of the offspring in the long term, either through in-utero contact or breastfeeding. $(2,3,4,5)$ In this study, the effects of gestation and breastfeeding were evaluated separately in $S$. mansoni-infected mothers. Therefore, the expression of HDACs, and cytokine production by lymphocytes and macrophages was assessed. These experiments were conducted using an in vitro system with broadly activated splenic cells, achieved using mitogenic stimulation.

Our findings show that breastfeeding from infected mothers induced the expression of HDACs from different classes which are involved in reducing the inflammatory response; however, gestation enhanced the expression of a single HDAC. These enzymatic changes induced by
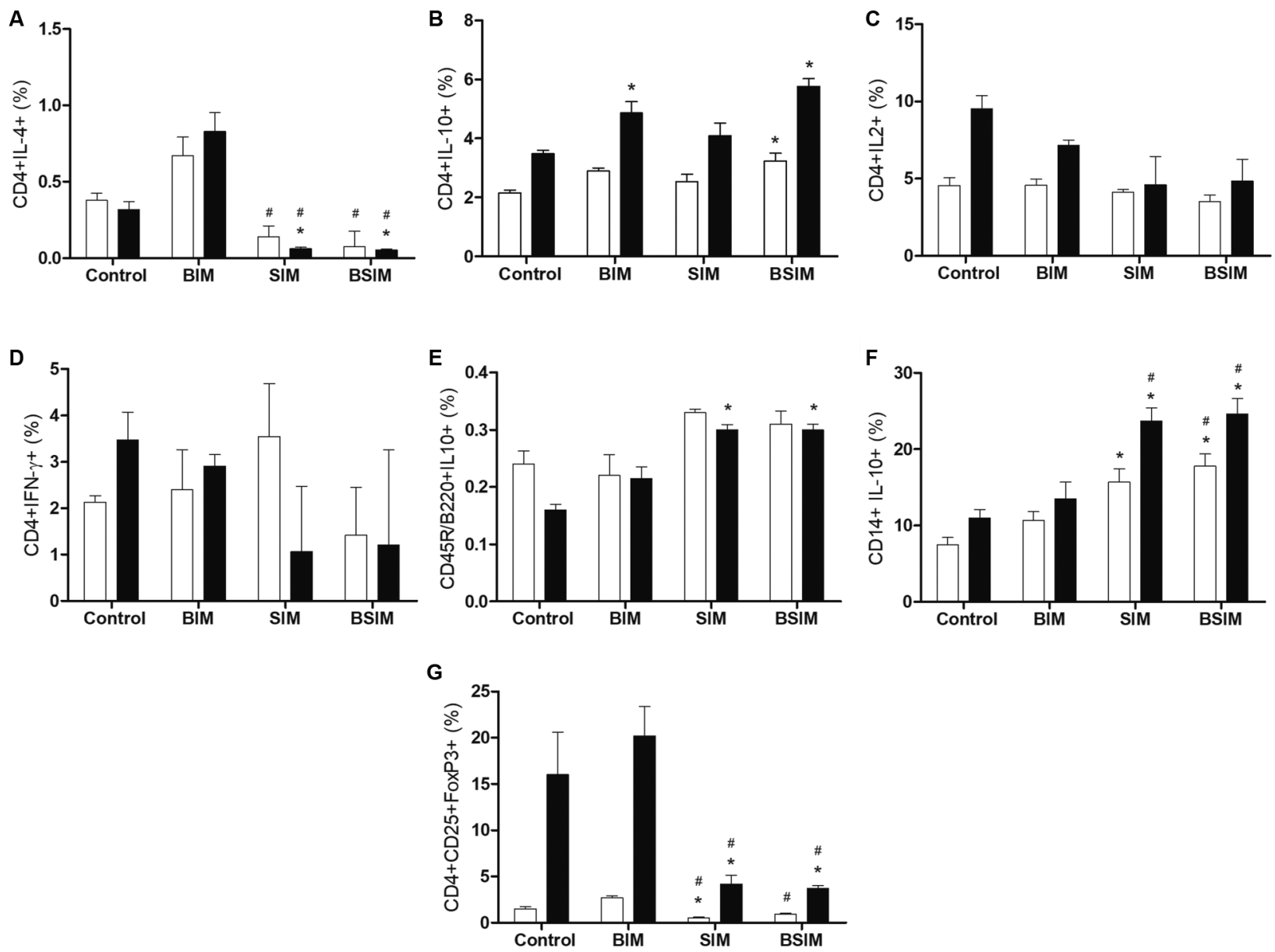

Fig. 7: cytokine production by $\mathrm{T}$ and $\mathrm{B}$ lymphocytes and monocytes, and splenocytes expressing CD4+ CD25+ FoxP3+. Frequency of CD4+IL-4+ (A), CD4+IL-10+ (B), CD4+IL-2+ (C), CD4+IFN- $\gamma+$ (D), CD45R/B220+IL-10+ (E), CD14+IL-10+ (F) and CD4+CD25+FoxP3+ (G) in splenocytes from Swiss Webster mice (seven weeks) born (BIM), breastfed (SIM) or born and breastfed (BSIM) in infected mothers, or uninfected mothers (Control), cultured for $24 \mathrm{~h}$ in the presence of mitogen (Concanavalin A) (5 $\mathrm{g} / \mathrm{mL}$, black bar) or with culture medium (BASAL, white bar). The frequencies were verified by flow cytometry. The results represent the median and standard error for $8-10$ animals/group. ${ }^{*} \mathrm{p}<0.05$ compared to control group. $\# \mathrm{p}<0.05$ compared to BIM and BSIM groups. 
breastfeeding or gestation appear to inhibit cells with a regulatory phenotype $(\mathrm{CD} 4+\mathrm{CD} 25+\mathrm{Fox} 3+)$, but favour an IL-10-dependent pathway.

Only offspring generated from infected mothers showed increased expression of HDAC9. According to Tao et al. ${ }^{(18)}$, HDAC9 is linked to decreased generation and performance of regulatory $\mathrm{T}$ cells. It has been observed that animals genetically deficient in HDAC9 had increased mRNA expression of Foxp3, CTLA-4, and GITR and increased CD4+FoxP3+ cells in lymphoid tissues. Regarding the cell phenotypes studied here, we observed that the generated offspring had a higher frequency of IL-10 producing CD4+ T cells. Together, these data suggest that immunomodulation in utero induced by maternal infection does not favour the generation of cells with a regulatory phenotype, but does favour IL-10 production via HDAC9 in offspring. In studies using immunisation with ovalbumin as adjuvant, gestation in infected mothers was correlated with increased production of IL$10 .^{(2,5)}$ Furthermore, Li et al. ${ }^{(19)}$ reported the positive regulation of HDAC9 in immature peritoneal macrophages through the action of acetyltransferase Dnmt3, leading to increased production of IFN- $\alpha$ and IFN- $\beta$ via deacetylation of kinase TBK1. Mice deficient for Dnmt3a showed greater susceptibility to infection by vesicular stomatitis virus (VSV). Thus, it is possible that gestation in schistosomotic mothers could alter the innate immune response against viral infections in offspring.

Prior exposure to the breast milk of schistosomotic mothers positively altered the expression of HDACs from different classes linked to reduction and/or resolution of the inflammatory response. ${ }^{(8,20,21)}$ There was an increase in the relative expression of HDACs 1,2 and 7, all of which show anti-inflammatory activity by inhibiting the transcription of NF- $\kappa \beta$ and decreasing the production of inflammatory cytokines (TNF- $\alpha$, IL-1, IL-6). $(7,22,23)$ There was a decrease in HDAC3 expression in all experimental groups (at baseline), but this recovered to levels similar to Control in response to mitogen.

HDAC6 and HDAC11 were also increased in animals that only received milk from infected mothers. Wang et al. ${ }^{(24)}$ when using an HDAC6 inhibitor, observed a reduction in HDCA6 recruitment to the IL-10 promoter along with enhanced TNF- $\alpha$, IL-12p 40 , and IFN- $\gamma$ production, as well as increased influx of macrophages, dendritic cells (DCs), and neutrophils to the lungs in response to Mycobacterium tuberculosis infection. In fact, Cheng et al. ${ }^{(6)}$ reported that HDAC6 is required for transcriptional activation of IL-10 gene expression in macrophages and dendritic cells through STAT3 activation, while HDAC11 acts as an IL-10 transcriptional repressor. Here, the expression of HDAC6 seemed to overlap with the repressive effect of HDAC11. These data are corroborated by the increased IL-10 production in macrophages (CD14+IL-10+) in the SIM group. It is worth noting that the increase in HDAC6 expression, together with the baseline HDAC5 decrease in breastfed animals, may be related to the decrease in Treg cell activity and markers (FoxP3, CTLA-4 and GITR). ${ }^{(25,26)}$ These data are corroborated by the lower frequency of CD4+CD25+FoxP3+ cells in animals which were breastfed under mi- togenic stimulus. Regarding the production of cytokines by $\mathrm{T}$ lymphocytes, decreased production of IL- 4 and IFN- $\gamma$ was observed (although there was no statistical difference for the latter). There was a subtle increase in the frequency of IL-10 producing B lymphocytes under mitogenic stimulation, and the baseline frequency was high in all study groups. These data corroborate the dependence of IL-10 on phagocytic cells in producing the suppressive and epigenetic effects of breast milk from schistosomotic mothers.

The high expression of HDAC10 was related to activation of the IL-1 $\beta$-mediated NF- $\kappa \beta$ signalling pathway in a study with mesenchymal stem cells derived from the synovial membrane in temporo-mandibular joint repair. (27) However, HDAC10 was shown to be important in regulating the production of reactive oxygen species (ROS) in gastric cancer, and its inhibition led to the accumulation of ROS, triggering the intrinsic apoptotic pathway. (28) It is known that ROS acts both in the innate and adaptive immune systems by attracting polymorpho-nuclear leukocytes, monocytes, and macrophages through Tolllike receptors (TLR). ${ }^{(29,30)}$ Here, there were increases in the expression of this enzyme in the group that received only milk (SIM) as well as in those that were born and breastfed (BSIM). This finding may be related to the activity of antioxidant enzymes in breast milk. ${ }^{(31)}$

Milk from infected mothers also led to increased expression of class III HDACs, Sirt2, and Sirt7. It is known that Sirt2 acts on the anti-inflammatory pathway in M2 macrophages through expression of Arginase 1 (ArgI) and $C d 11 c,{ }^{(21)}$ and reduces ROS by increasing superoxide dismutase 2 (SOD2), catalase, and glutathione peroxidase. ${ }^{(8)}$ The increase in Sirt2 was related to inhibition of the NF- $\kappa \beta$ pathway and reduced expression of IL-1 $1 \beta$, IL-6, and TNF- $\alpha .{ }^{(21)}$ Sirt7 acts on the stabilisation of TGF- $\beta$ receptor type 1, allowing for efficient signalling of TGF- $\beta .^{(32)}$ This targeting to an anti-inflammatory response profile is consistent with the tolerogenic effect of breast milk. ${ }^{(33)}$ According to our data, offspring who were only breastfed by schistosomotic mothers and who had undergone postnatal infection by $S$. mansoni also had elevated levels of TGF- $\beta$, in addition to decreased nitric oxide production (Unpublished observations).

High expression of Sirt6 was observed in all experimental groups (born and/or breastfed animals). According to Lee et al., (20) high expression of Sirt6 acts to inhibit the NF- $\kappa \beta$ pathway and blocks the effect of TNF- $\alpha$. Li et al. ${ }^{(34)}$ showed that overexpression of Sirt6 inhibits RIGI-like receptor (RLR) and Toll-like receptor 3 (TLR3) in Dengue virus (DENV) infection, and the sirtuin core domain of SIRT6 is required for the inhibition of NF- $\mathrm{KB}$ p65 function, negatively regulating DENV-induced inflammatory responses via the RLR and TLR3 signalling pathways. These observations support the inhibition of inflammation we observed in the offspring of schistosomotic mothers.

The high expression of Sirt5 was also observed in the SIM group. It has been shown that Sirt5 is responsible for deacetylating STAT3, disrupting its activity at the mitochondrial level..$^{(35)}$ Qin et al. ${ }^{(36)}$ demonstrated that deficiencyof this enzyme led to a decrease in the 
innate inflammatory response, with lowered production of IL- 6 , TNF- $\alpha$ and, MCP-1 both in the hyper inflammatory and hypo inflammatory stages of sepsis in an animal model. In addition, it was observed that high expression of Sirt5 leads to an increased pro-inflammatory response by decreasing the interaction between Sirt2 and NF- $\kappa \beta p 56 .{ }^{(36)}$ Here, there was no decrease in Sirt2, and the increase in Sirt6 and Sirt7 may have overlapped with the effect of Sirt5.

Thus, HDACs were epigenetically altered by maternal infection and were involved in the suppression of pro-inflammatory mediators and increased IL-10 production, but did not favour cells with a regulatory phenotype. Even knowing the importance of cell-cell interactions in both in vivo and in vitro systems, it would be important to culture purified cells to understand if this pattern of expression of HDACs, indicating a predisposition to IL-10 dependent anti-inflammatory effects, was due to previous programming of $\mathrm{T}$ lymphocytes and macrophages in the BIM and SIM groups, respectively.

In conclusion, gestation in infected mice led to increased expression of HDAC9 alone, while breastfeeding from schistosomotic mothers led to increased expression of HDACs of all classes. It is true that the extrapolation of these data from mice to humans should be carefully evaluated, but our findings highlight the importance of experimental and clinical approaches to investigate the efficacy of therapeutic targets in allergy, autoimmunity, and cancer models after long-term epigenetic changes result in offspring from areas endemic for schistosomiasis. Therefore, our results shed light on immunological factors resulting from infection during early age, caused by modifications in epigenetic profile. In view of this, it will be important to evaluate factors in breast milk of infected mothers and how these components may modify the epigenetic and immunological profile of offspring.

\section{ACKNOWLEDGEMENTS}

To Gerlane Tavares de Souza Chioratto for veterinary support, Dr Danyelly Bruneska Gondim Martins for qPCR support, and PDTIS/Fiocruz, for the use of its facilities.

\section{AUTHORS' CONTRIBUTION}

GCRH, VMOS and JLLF - Conceived and designed the proposal; GCRH, MCS and FOS - performed lab experiments and processed the data; VMBL, FOS and MCPAA - contributed reagents/materials/analysis/tools; GCRH - writing - original draft; GCRH, VMOS, FOS, VMAC and JLLF - writing, review and editing; JLLF and VMOS - supervision. The authors declare no conflicts of interest.

\section{REFERENCES}

1. Salawu OT, Odaibo AB. Schistosomiasis among pregnant women in rural communities in Nigeria. Int J Gynaecol Obstet. 2013; 122(1): 1-4.

2. dos Santos P, Sales IR, Schirato GV, Costa VM, Albuquerque MC, Souza VM, et al. Influence of maternal schistosomiasis on the immunity of adult offspring mice. Parasitol Res. 2010; 107(1): 95-102.

3. dos Santos P, Lorena VM, Fernandes E, Sales IR, Albuquerque MC, Gomes Y, et al. Maternal schistosomiasis alters costimulatory molecules expression in antigen-presenting cells from adult offspring mice. Exp Parasitol. 2014; 141: 62-7.
4. Straubinger K, Sabine P, Costa OP, Ritter M, Buch T, Busch DH, et al. Maternal immune response to helminth infection during pregnancy determines offspring susceptibility to allergic airway inflammation. J Allergy Clin Immunol. 2014; 134(6): 1271-9.

5. Fernandes ES, Lorena VMB, Sales IRF, Albuquerque MCPA, Gomes YM, Costa VMA, et al. Maternal schistosomiasis: IL-2, IL-10 and regulatory $\mathrm{T}$ lymphocytes to unrelated antigen in adult offspring mice. Rev Soc Bras Med Trop. 2018; 51(4): 546-9.

6. Cheng F, Lienlaf M, Perez-Villarroel P, Wang HW, Lee C, Woan K, et al. Divergent roles of histone deacetylase 6 (HDAC6) and histone deacetylase 11 (HDAC11) on the transcriptional regulation of IL10 in antigen presenting cells. Mol Immunol. 2014; 60(1): 44-53.

7. Dahllöf MS, Christensen DP, Harving M, Wagner BK, MandrupPoulsen T, Lundh M. HDAC inhibitor-mediated beta-cell protection against cytokine-induced toxicity is STAT1 Tyr701 phosphorylation independent. J Interferon Cytokine Res. 2015; 35(1): 63-70.

8. Kosciuczuk EM, Mehrotra S, Saleiro D, Kroczynska B, MajchrzakKita B, Lisowski P, et al. Sirtuin 2-mediated deacetylation of cyclin-dependent kinase 9 promotes STAT1 signaling in type I interferon responses. J Biol Chem. 2019; 294(3): 827-37.

9. Attig L, Gabory A, Junien C. Early nutrition and epigenetic programming: chasing shadows. Curr Opin Clin Nutr Metab Care. 2010; 13(3): 284-93.

10. Brand S, Teich R, Dicke T, Harb H, Yildirim AÖ, Tost J, et al. Epigenetic regulation in murine offspring as a novel mechanism for transmaternal asthma protection induced by microbes. J Allergy Clin Immunol. 2011; 128(3): 618-25.e1-7.

11. Song Y, Liu C, Hui Y, Srivastava K, Zhou Z, Chen J, et al. Maternal allergy increases susceptibility to offspring allergy in association with TH2-biased epigenetic alterations in a mouse model of peanut allergy. J Allergy Clin Immunol. 2014; 134(6): 1339-45.

12. Kouzarides T. Chromatin modifications and their function. Cell. 2007; 128(4): 693-705.

13. Haberland M, Montgomery RL, Olson EN. The many roles of histone deacetylases in development and physiology: implications for disease and therapy. Nat Rev Genet. 2009; 10(1): 32-42.

14. Yang Z, Wang T, Wang F, Niu T, Liu Z, Chen X, et al. Discovery of selective histone deacetylase 6 inhibitors using the quinazoline as the cap for the treatment of cancer. J Med Chem. 2015; 59(4): $1455-70$.

15. Stolfa DA, Marek M, Lancelot J, Hauser AT, Walter A, Leproult E, et al. Molecular basis for the antiparasitic activity of a mercaptoacetamide derivative that inhibits histone deacetylase 8 (HDAC8) from the human pathogen Schistosoma mansoni. J Mol Biol. 2014; 426(20): 3442-53.

16. Vishwakarma S, Iyer LR, Muley M, Singh PK, Shastry A, Saxena A, et al. Tubastatin, a selective histone deacetylase 6 inhibitor shows anti-inflammatory and anti-rheumatic effects. Int Immunopharmacol. 2013; 16(1): 72-8.

17. Katz N, Chaves A, Pellegrino J. A simple device for quantitative stool thick-smear technique in schistosomiasis mansoni. Rev Inst Med Trop Sao Paulo. 1972; 14(6): 397-400.

18. Tao X, Yan Y, Lu L, Chen B. HDAC10 expression is associated with DNA mismatch repair gene and is a predictor of good prognosis in colon carcinoma. Oncol Lett. 2017; 14(4): 4923-9.

19. Li X, Zhang Q, Ding Y, Liu Y, Zhao D, Zhao K, et al. Methyltransferase Dnmt3a upregulates HDAC9 to deacetylate the kinase TBK1 for activation of antiviral innate immunity. Nat Immunol. 2016; 17(7): 806-15.

20. Lee HS, Ka SO, Lee SM, Lee SI, Park JW, Park BH. Overexpression of sirtuin 6 suppresses inflammatory responses and bone destruction in mice with collagen-induced arthritis. Arthritis Rheum. 2013; 65(7): 1776-85. 
21. Lo Sasso G, Menzies KJ, Mottis A, Piersigilli A, Perino A, Yamamoto $\mathrm{H}$, et al. SIRT2 deficiency modulates macrophage polarization and susceptibility to experimental colitis. PLoS One. 2014; 9(7): e103573.

22. Ito K, Yamamura S, Essilfie-Quaye S, Cosio B, Ito M, Barnes PJ, et al. Histone deacetylase 2-mediated deacetylation of the glucocorticoid receptor enables NF-kappaB suppression. J Exp Med. 2006; 203(1): 7-13.

23. Barneda-Zahonero B, Román-González L, Collazo O, Rafati H, Islam AB, Bussmann LH, et al. HDAC7 is a repressor of myeloid genes whose downregulation is required for transdifferentiation of pre-B cells into macrophages. PLoS Genet. 2013; 9(5): e1003503.

24. Wang X, Tang X, Zhou Z, Huang Q. Histone deacetylase 6 inhibitor enhances resistance to Mycobacterium tuberculosis infection through innate and adaptive immunity in mice. Pathog Dis. 2018; 1: 76(6).

25. de Zoeten EF, Wang L, Butler K, Beier UH, Akimova T, Sai H, et al. Histone deacetylase 6 and heat shock protein 90 control the functions of Foxp3(+) T-regulatory cells. Mol Cell Biol. 2011; 31(10): 2066-78.

26. Xiao H, Jiao J, Wang L, O’Brien S, Newick K, Wang LC, et al. HDAC5 controls the functions of Foxp3(+) T-regulatory and CD8(+) T cells. Int J Cancer. 2016; 138(10): 2477-86.

27. Liao W, Sun J, Liu W, Li W, Jia J, Ou F, et al. HDAC10 upregulation contributes to interleukin $1 \beta$-mediated inflammatory activation of synovium-derived mesenchymal stem cells in temporomandibular joint. J Cell Physiol. 2019; 234(8): 12646-62.

28. Lee JH, Jeong EG, Choi MC, Kim SH, Park JH, Song SH, et al. Inhibition of histone deacetylase 10 induces thioredoxin- interacting protein and causes accumulation of reactive oxygen species in SNU-620 human gastric cancer cells. Mol Cells. 2010; 30(2): 107-12

29. Hattori H, Subramanian KK, Sakai J, Jia Y, Li Y, Porter TF, et al. Small-molecule screen identifies reactive oxygen species as key regulators of neutrophil chemotaxis. Proc Natl Acad Sci USA. 2010; 107(8): 3546-51

30. Martinon F, Chen X, Lee AH, Glimcher LH. TLR activation of the transcription factor XBP1 regulates innate immune responses in macrophages. Nat Immunol. 2010; 11(5): 411-8.

31. Castillo-Castañeda PC, García-González A, Bencomo-Alvarez AE, Barros-Nuñez P, Gaxiola-Robles R, Méndez-Rodríguez LC, et al. Micronutrient content and antioxidant enzyme activities in human breast milk. J Trace Elem Med Biol. 2019; 51: 36-41.

32. Yamagata K, Yoshizawa T. Transcriptional regulation of metabolism by SIRT1 and SIRT7. Int Rev Cell Mol Biol. 2018; 335: 143-66.

33. Blewett HJH, Cicalo MC, Holland CD, Field CJ. The immunological components of human milk. Adv Food Nutr Res. 2008; 54: 45-80.

34. Li P, Jin Y, Qi F, Wu F, Luo S, Cheng Y, et al. SIRT6 acts as a negative regulator in dengue virus-induced inflammatory response by targeting the DNA binding domain of NF- $\kappa \mathrm{B}$ p65. Front Cell Infect Microbiol. 2018; 8: 113.

35. Xu YS, Liang JJ, Wang Y, Zhao XJ, Xu L, Xu YY, et al. STAT3 undergoes acetylation-dependent mitochondrial translocation to regulate pyruvate metabolism. Sci Rep. 2016; 6: 39517.

36. Qin K, Han C, Zhang H, Li T, Li N, Cao X. NAD+ dependent deacetylase Sirtuin 5 rescues the innate inflammatory response of endotoxin tolerant macrophages by promoting acetylation of $\mathrm{p} 65$. J Autoimmun. 2017; 81: 120-9. 